\title{
A peer pressure experiment: Recreation of the Asch conformity experiment with robots.
}

\author{
Jürgen Brandstetter ${ }^{1}$, Péter Rácz ${ }^{2}$, Clay Beckner ${ }^{2}$, Eduardo B. Sandoval ${ }^{1}$, Jennifer Hay ${ }^{2}$, Christoph Bartneck ${ }^{1}$
}

\begin{abstract}
The question put forward in this paper is whether robots can create conformity by means of group pressure. We recreate and expand on a classic social psychology experiment by Solomon Asch, so as to explore three main dimensions. First, we wanted to know whether robots can prompt conformity in human subjects, and whether there is a significant difference between the degree to which individuals conform to a group of robots as opposed to a group of humans. Secondly we ask whether group pressure (from human or robot peers) can exert influence in verbal judgments, analogously to the influence on visual judgments that is known from previous research [3], [2]. Thirdly, we investigate whether the level of conformity differs between an ambiguous situation and a non-ambiguous situation.

Our results show that in both visual and verbal tasks, participants exhibit conformity with human peers, but not with robot peers. The social influence of robot peers is not a significant predictor of verbal or visual judgments in our tasks. Furthermore, the level of conformity is significantly higher in an ambiguous (unclear) situation.
\end{abstract}

\section{INTRODUCTION}

When we look at the current development of humanlike social robots, it is possible to predict a future where robots help out in the office, teach children in the classroom, become companions, work in advertising, or help in our households [5], [14], [20]. During the last few years, there has been an observable trend towards service robots outflanking industrial robots in production volume. Whereas all sold industrial robots total 2.3 million units, more than 2.5 million units of service robots were sold in 2011 alone, with an estimated 16 million further units to be sold between 2012 and 2015 [24]. The importance of robots is not only recognized by the science community and the industry, but also by governments. For example, the New Zealand government has put the development of robots on their main future agenda.

With this increased presence of robots in domestic life, the question remains as to how these robots will be regarded by humans-as mindless machines, as subservients, or as peers? Previous work demonstrates that humans treat computers socially, for instance, by tempering unpleasant feedback to a computer, apparently so as to be more polite [19], [9]. Such findings raise the question of whether it is possible for robots to prompt human conformity.

\footnotetext{
${ }^{1}$ Human Interface Technology Lab, University of Canterbury, P.O. Box 4800, Christchurch, 8140, New Zealand

${ }^{2}$ New Zealand Institute of Language, Brain and Behaviour, University of Canterbury, P.O. Box 4800, Christchurch, 8140, New Zealand

Please direct correspondence to Jürgen Brandstetter at juergen.brandstetterapg.canterbury.ac.nz or Péter Rácz at peter.racz@canterbury.ac.nz
}

To investigate the persuasive power of robots, we expand on two landmark psychology experiments. The first experiment was conducted by Muzafer Sherif, who studied a conformity effect in an ambiguous situation. The experiment builds on the autokinetic effect, a phenomenon in which a person perceives a sudden movement of a light point when no reference is given. Sherif created this effect by sitting participants in a dark room with one small light point visible. The participants had to look at this point, and had to say how much the light point moved. However, the light never actually moved, but the autokinetic effect created an illusory sudden movement. This movement is perceived differently by different people. After he did the experiment with one person, Sherif set two or three people in the same room and asked them to say out loud how much the point moved. Astonishingly, after three rounds all the participants said the same number, even though everyone perceived a different movement. This effect is called informational conformity or social proof and describes the effect in which people in an uncertain situation look at their neighbors to see what is probably the right answer and conform with them. [7], [21], [15].

A second experiment is reported by Solomon Asch in his influential paper "Effects of group pressure upon the modification and distortion of judgements". Asch builds on the findings of Sherif to find out whether humans also conform in non-ambiguous situations. His experiment simulated a simple visual line test. The participant saw three lines of different heights, labelled A, B, C on the left side of a board, and one reference line on the right side labelled with "?" (see Figure 1). The task was to say which line matched the reference line. When the participants were alone in a room to perform the test they almost always gave the correct answer. In a second round Asch placed the participants in the same room with other participants who were all actors and who all gave the wrong answer. Even though the real participant - presumably - knew the correct answer, in $32 \%$ of all tasks the participants went along with the group if the group size was bigger than four. In this case, Asch provided evidence that conformity is not only influential in ambiguous situations but also in non-ambiguous ones [2], [3], [4].

In the current study, we recreate the Asch experiment and use aspects of the Sherif experiment as a model for further research. We investigate a visual judgment task along the lines of Asch [2], [3], [4], and extend the methodology to a verbal production task. Verbal tasks may provide an especially fruitful domain for conformity studies, because language is inherently social, and speakers influence one 


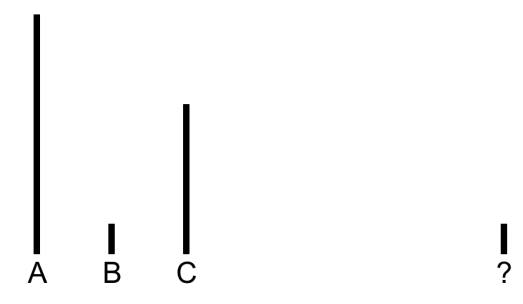

Fig. 1: An example of the lines task, where the participants have to identify the corresponding line. Based on Solomon Asch's experiment.[2]

\section{think lose leave make bring}

Fig. 2: An example of a word set presented in the word task. The four confederates each produce the past tense form of the first four verbs. Participants then have to provide the correct past tense form of the fifth

another's linguistic behaviors on an ongoing basis. The perceived social characteristics of a speaker seem to play a role in speech processing, and the social status of speakers affects processes of language change[12], [13]. Moreover, it is known that speakers tend to copy one another in natural settings; individuals align their actions and representations, interactively and subconsciously [10]. Speakers have many choices available when they speak; for instance they may often choose from different pronunciations of the same word. In a conversational context, one speaker's choice of a particular linguistic variant typically increases the likelihood that other speakers will choose the same variant. In most cases, speakers accommodate to each other, and their linguistic styles "converge", as is found by observing many linguistic domains including speech rate [22], word choices [6], and choices of grammatical constructions [23]. Speaker convergence is subject to social variables; speakers tend to accommodate to one another unless they want to establish social distance from their interlocutor(s) [1], [17], [18].

In the current experiment, we explore a form of linguistic convergence between speakers with respect to morphological variation, specifically involving the English past tense. In some cases speakers may choose between various past tense patterns, as in "dived" or "dove" for the past tense of "dive", or "slayed" or "slew" as the past tense of "slay". Such morphological pairs are known as morphological "doublets"; individual speakers may alternate between different choices in different circumstances [11], and some competing morphological items have co-existed side-by-side for centuries [8]. For instance, the Oxford English Dictionary indicates that the past-tense forms "leaped" and "leapt" have coexisted in English since at least the 1500s, as have "dreamed" and "dreamt". Since no single past tense form is universally preferred, morphological doublets offer a verbal analogue to ambiguous visual judgements. The proposal of the current study is that the choice of one verb variant over another is a behavior that may be influenced by other speakers. That is, speakers who influence one another socially should be expected to converge on the same past tense form (or the same past tense verb pattern). Building on the above experiments and our interests in Human Robot Interaction and verbal communication, we developed an experiment which includes the following conditions: ambiguous and non-ambiguous line conditions, and ambiguous and nonambiguous verbal conditions. We ran these conditions with four confederate robots (for one group of participants) or four confederate human actors (for the other). Data in the peer conditions is compared against matched baseline data, gathered from participants in isolation. The first question we wanted to answer was whether participants conform more (or less) to a robot group than to a human group. The second question is whether conformity to verbal judgments can be observed in a peer-pressure experiment, comparable to the classic conformity studies by Asch. In the last question we wanted to understand whether participants conform more to the peer group in an ambiguous task than in a non-ambiguous task.

For clarity, we summarize our questions here again:

Q1: Do participants conform more to the human group than the robot group?

Q2: Do participants conform to a group's verbal judgments in addition to visual judgments?

Q3: Do participants conform more to the the group in an ambiguous task than in an non-ambiguous one?

\section{Methods}

We performed a $2 \times 2 \times 2$ mixed-factor experiment. The within-subject variables were the modality of the stimulus (lines vs. words), the ambiguity of the stimulus (ambiguous vs. non-ambiguous), and the ordering of the stimuli (lines first vs. words first). The across-subject variable was the setting: a group of human peers, a group of robot peers, or alone (the baseline). The number of times the participants conformed to their peers was the dependent variable. In the baseline setting, an error in the visual task was the dependent variable, and in the verbal task the selection of the regular (-ed-final) past tense form was the dependent variable. Such responses match the group behaviors in the peer conditions, as described below, and thus their occurrence in isolation provides us with information about baseline behavior.

\section{A. Design}

We aimed to re-create the Asch and Sherif experiments introduced above, expanding the visual decision task with a verbal task and the group of human confederates with a group of robots. 
The line projection consisted of three horizontal parallel lines labelled A, B, C on the left side of the projection and one line labelled with ? on the right side. An example can be seen in Figure 1. The line on the right, marked with a ? matches one of the lines on the left. The task for the participant was to say which line, A, B, or C, matched the line labelled ?, following the model of Asch's experiment.

The word projection consisted of groups of five words. The task was to say the word on the projection and its past tense form, for example like and liked. Each participant had to say the word corresponding to their position. This means participant one would say the first word and its past tense, participant two would say the second word and its past tense, and so on. An example word set is given in Figure 2.

Each experiment block (involving either lines or words) was divided into three parts: a warmup (3 subject responses), an ambiguous-item set (15 subject responses), and a nonambiguous-item set (15 subject responses). Each experiment block thus consisted of 33 trials, although warmup items are not analyzed, leaving 30 responses of interest in each block, per subject.

In the warmup part, consisting of three unambiguous tasks, the peer group would say the correct answer-thus providing the correct visual judgment in the lines task, or providing the normative past tense in the verbal task. In this case we wanted to give the participants the feeling that the robots/actors understood the question, and could provide correct responses.

In the second and third parts of each experiment block, the experiment confederates (robots or human actors) gave consistent answers, with the intention of swaying participants' responses away from their default choices. In the visual task, the confederate actors/robots would always choose the incorrect line response. In the verbal task, the confederates always provided the regular past tense form, that is, a form which ends in -ed.

In the verbal task, 'ambiguous' items are stimulus verbs for which variation exists in English usage, and the target words can have a regular or irregular past tense form, for example, dive - dived, or dive - dove. Non-ambigous verbal stimuli are items which have only an irregular past tense form, for example, run-ran. Note that this means that in the peer-group conditions, the robots/actors all provided verb forms which are not normatively in use, that is, overregularized forms such as runned as the past tense of run.

Additional details are provided in the 'Baseline' section below regarding about the designation of ambiguous/nonambiguous items in the visual task.

We counter-balanced the order of the line and word experiment blocks. We used pre-recorded introductions in all conditions.

\section{B. Apparatus}

The setting consisted of a projector, a high quality wireless microphone, a table with five chairs, four customized Aldebaran NAO robots or four confederate actors, and a laptop to control the recordings and experiment. The projecting area

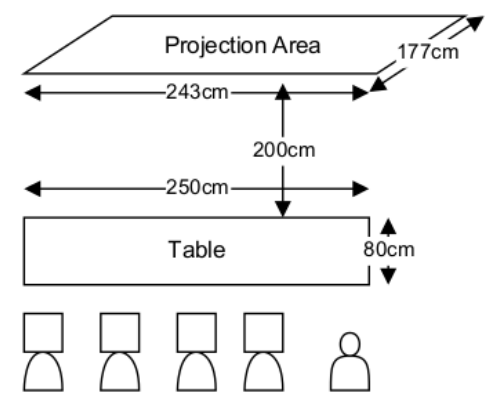

Fig. 3: Plan of the physical setting of the test chamber.

had a dimension of $243 \times 177 \mathrm{~cm}$, although the maximum line length was $118 \mathrm{~cm}$. The table was exactly parallel to the projecting area at a distance of $200 \mathrm{~cm}$. The dimensions of the table were $80 \times 250 \mathrm{~cm}$ (see Figure 3 ).

To make our NAO robots more individualistic, we gave every robot different outfits and voices. We did this individualization process on the assumption that if robots were distinctive, participants would more easily see them as individuals rather than as copies of each other. Since we wanted to make sure that the robots' appearance rather than their voices constitutes the only difference between the actors and the robots, we used recordings of the actors for the robot voices. We also made sure that each robot (in the robot condition) sat in the position corresponding to the actor (in the human condition) whose voice it shared.

\section{Procedure}

So as to make the human and robot conditions as consistent as possible, the start of the experiment was designed so as to discourage spontaneous interactions between the participant and the confederates. In both conditions, the participant signed consent forms in a room separate from the experiment room. The participant was then informed that the other participants were already in the experiment room and the study was ready to begin. When the participant entered the second room, all of the confederates greeted him or her (with a simple 'Hello', 'Hi there,' etc.) In the robot condition, the robot confederates turned their heads toward the participant when making the greeting, to give a sense that the robots were aware of the participant's presence. The research assistant told the participant to find an empty chair; since the confederates were already seated, the participant had no option but to take the last chair in the row (See Fig. 3). After the participant sat down, the experiment immediately started.

\section{Baseline}

We ran two baseline studies to provide reference points for judgments in the visual and verbal tasks, in the absence of any peer pressure.

The baseline setting for the visual task consisted of 107 line configurations. The setup of the room was exactly the same as in the group experiment, the only difference being that the participants did not say out loud which line best 
matched; they used the buttons $1,2,3$ on the keyboard. We assume there is no difference between saying the answer out loud or pressing a button when no one is in the room other than the participant. Out of these 107 settings, we picked exactly 30 for our group experiment. The corpus of 30 lines was divided into two parts. The first part (15 settings) contained all the settings where people made most of the mistakes. These lines were ambiguous and not easy to distinguish. The second part of the lines contained a set of lines where the two closest lines had no difference bigger than $30 \%$ and people made only one or zero mistakes. We chose this setting because Asch used a similar setting to prove his hypothesis [2]. We used baseline responses to these 30 targets as a comparison to the group settings.

The baseline setting for the verbal task was the same as the group setting, except that the participant had to produce past tenses for all the forms on the screen. All items that were presented in the peer conditions (to confederates as well as participants) were included in the baseline, to control for possible priming effects. However, for purposes of quantitative comparison between baseline and peer conditions, we only analyze the 30 verbs for which participants in the peer groups gave responses. We used consistent coding for the baseline and the group settings, labeling only regularized responses as 'conforming'.

Unlike the group settings, the baseline settings for the lines and the words were run with two different sets of participants.

\section{E. Participants}

We had 78 total participants; 23 participated in the group of human confederates, 19 in the group of robots, 18 in the lines baseline, and 18 in the verbal baseline.

All participants were college students at the University of Canterbury, and all were native speakers of New Zealand English. It was important to use speakers from one dialect region only, as there may be regional variation in preferences for past tense forms.

No participants reported any previous familiarity with the Asch experiment during debriefing. All participants were paid $\$ 10$ for their participation. The experiment lasted about 20-30 minutes.

\section{RESULTS}

Conformity rates for all conditions are summarized in Figure 4.

Inspection of this figure reveals a few interesting findings, starting with the baseline regularization of verbs 'conformity' in the verbal task when there are no peers to conform to. Note that among non-ambiguous verbs, $5 \%$ of baseline participants' responses were in fact regularized. Such responses represent spontaneous over-regularizations of verbs which are not expected to deviate from their irregular status: find-finded, go-goed, know-knowed. Thus, the regularizations in the baseline verbal task are markedly more common than in written corpus data, based on searches of large online print corpora such as the Google Books

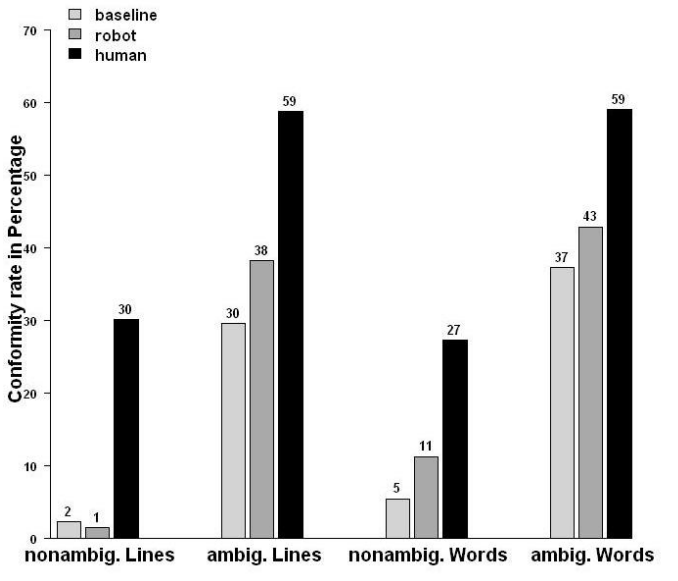

Fig. 4: Percentage conformity for all conditions. The 'conformity' in the baseline conditions represents the behavior of interest (error in the lines task, and regularization in the verbal task) by isolated subjects, and is thus used as a reference point for matched items in the robot and human peer conditions.

\begin{tabular}{|c|c|c|c|c|}
\hline & coef & st error & Z & sig \\
\hline Intercept (baseline) & -5.46 & 0.58 & -9.396 & $* * *$ \\
\hline group=humans & 3.91 & 0.74 & 5.267 & $* * *$ \\
\hline group=robots & 0.55 & 0.83 & 0.668 & n.s. \\
\hline ambiguous $=\mathrm{T}$ & 4.57 & 0.54 & 8.403 & $* * *$ \\
\hline group $=$ humans $\mathrm{x}$ ambiguous $=\mathrm{T}$ & -2.51 & 0.65 & -3.889 & $* * *$ \\
\hline group=robots $\mathrm{x}$ ambiguous $=\mathrm{T}$ & -0.18 & 0.73 & -0.248 & n.s. \\
\hline
\end{tabular}

TABLE I: Mixed Effects Model Summary

corpus [16]. This verifies that for purposes of quantitative comparison, a laboratory baseline study was essential for interpreting our peer-group results. Production of a sequence of English past tense forms is an admittedly artificial task, whether performed in the presence of peers or in isolation, and speakers may also prime themselves to regularize verbs.

In all conditions, there is a clear and strong effect of conformity induced by the human peer group. In three of the four conditions, the robots appear to elicit slightly higher rates of the conforming judgements than the baseline data, although the rates are markedly lower than for the humans.

However, in statistical analyses, we do not find evidence of significant effects of peer influence by robots. A binomial mixed-effects model was stepwise fit on the data using maximal random effect structure. Non-significant effects were dropped from the model. The model summary is presented in Table I. For purposes of economy, we only present the overall model here, which encompasses conformity in both visual and verbal tasks as the dependent variable. However, separate models fit independently to the lines and verbs datasets also show the same pattern of main effects for peer group (human or robot) and ambiguity.

The mean per-subject conformity rates for the relevant across-subject and within-subject settings can be seen in 
Figure 5. The degree of conforming responses is significantly higher for humans than for robots and baseline. Robots and baseline are not significantly different. This is true for both the ambiguous and the non-ambiguous settings. The degree of conforming responses are significantly higher for the ambiguous setting than for the non-ambiguous setting. This difference is significantly larger for the human group. Whether lines come first is not significant. Neither is task type (lines or words).

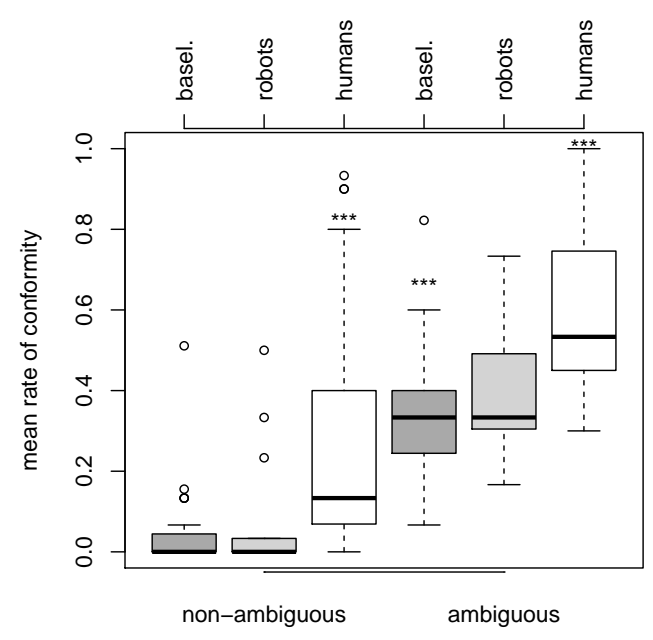

Fig. 5: Subject means for group setting and ambiguity: both line and word tasks

\section{COnClusions/Discussion}

In this study, our main goal was to find out if robots could create conformity and if so, how much difference there is between human peers and robot peers. The study uses the idea from Sherif, who studied conformity in ambiguous situations and Asch, who studied conformity in non-ambiguous situations.

In our first question (Q1) we wanted to know if robot peers elicit the same conformity as human peers. The analyses point to a clear null result with respect to peer influence from robots. When surrounded by robot peers, individuals' behavior shows some trend toward being influenced in the anticipated direction, but this behavior is not significantly different from the baseline behavior in isolation from peers. Subjects' behavior alongside robot peers patterns more closely with the baseline condition than it does with the human-peer condition.

Of course, null results are always more difficult to interpret than positive results. One might call into question various features of our setup: it is always possible significant results could arise if the sample size were larger, if the robots looked or acted more human-like, or if the experimental instructions were different. Additional investigation in these areas would certainly be appropriate. We should reiterate, however, that significant results are evident across the board in our study when the peers are human confederates. Moreover, our analyses demonstrate that participants' behavior among robot peers is significantly different from behavior among human peers. Individuals clearly interpret robot peers differently from human peers.

In our second question (Q2) we wanted to know if participants conform to a group in verbal tasks as well as in visual tasks. Our data indicates that individuals are susceptible to social pressure in choosing past tense forms - again, when the peers are humans, although not when they are robots. This result supplements a wide range of findings that speakers interactively align during dialogues, adding morphological convergence to the inventory of different linguistic levels for which convergence has been observed.

In our third question (Q3) we wanted to know if there is more conformity in an ambiguous condition versus a non-ambiguous situation. The ambiguous situation is often referred to as social proof [7]. Social proof means that when people are in an uncertain situation, they tend to look around to see what their peers do. Conformity is clearly higher in ambiguous cases, as verified by the regression model.

\section{A. Future Work}

There are many aspects of this work that warrant further investigation. We are particularly interested in conducting further work into understanding individual variation across participants, and the types of peer pressure that different individuals may be vulnerable to. We also intend to attempt a conformity task which is closer to "natural" interaction, in which participants are not just giving responses in isolation.

\section{ACKNOWLEDGMENTS}

This project was made possible through the support of a Sub-award under a grant to Northwestern University from the John Templeton Foundation (Award ID 36617). The opinions expressed in this publication are those of the author(s) and do not necessarily reflect the views of the John Templeton Foundation. Hay and Beckner were also supported by a Rutherford Discovery Fellowship awarded to Hay.

This project was approved by the University Canterbury Ethics Committee: HEC APPLICATION 2013/111 and the Northwestern University Ethics Committee: STU00085449.

A special thanks goes to the four actors; Timothy Pomroy, Natalie Eustace, Julian Melchert, Morgana Mountfort-Davies and to Latifah Almanea for recruiting.

\section{REFERENCES}

[1] C. Abrego-Collier, J. Grove, M. Sonderegger, and A. C. L. Yu. Effects of speaker evaluation on phonetic convergence. In Proc. Int. Congress of the Phonetic Sciences, Hong Kong, pages 192-195, 2011.

[2] S. E. Asch. Effects of group pressure upon the modification and distortion of judgments. Groups, Leadership, and Men., pages 222236, 1951.

[3] S. E. Asch. Opinions and social pressure. Scientific American, pages 31-35, 1955.

[4] S. E. Asch. Studies of independence and conformity: I. A minority of one against a unanimous majority. Psychological Monographs: General and Applied, 70(9):1-70, 1956.

[5] C. L. Breazeal. Designing sociable robots. MIT press, 2004. 
[6] S. E. Brennan and H. H. Clark. Conceptual pacts and lexical choice in conversation. Journal of Experimental Psychology: Learning, Memory, and Cognition, 22(6):1482, 1996.

[7] R. B. Cialdini. Influence : science and practice. HarperCollins, 2009.

[8] C. Fehringer. How stable are morphological doublets? A case study of schwa/Ø variants in Dutch and German. Journal of Germanic linguistics, 16(4):285-329, 2004.

[9] B. J. Fogg. Persuasive technology. Ubiquity, 2002(December):2, Dec. 2002.

[10] S. Garrod and M. J. Pickering. Joint action, interactive alignment, and dialog. Topics in Cognitive Science, 1(2):292-304, 2009.

[11] L. R. Haber. Leaped and Leapt: a theoretical account of linguistic variation. Foundations of Language, 14(2):211-238, 1976.

[12] J. Hay, P. Warren, and K. Drager. Factors influencing speech perception in the context of a merger-in-progress. Journal of Phonetics, 34(4):458-484, Oct. 2006.

[13] D. J. Hruschka, M. H. Christiansen, R. A. Blythe, W. Croft, P. Heggarty, S. S. Mufwene, J. B. Pierrehumbert, and S. Poplack. Building social cognitive models of language change. Trends in Cognitive Sciences, 13(11):464-9, Nov. 2009.

[14] T. Kanda, T. Hirano, D. Eaton, and H. Ishiguro. Interactive robots as social partners and peer tutors for children: A field trial. Humancomputer interaction, 19(1):61-84, 2004.

[15] S. A. McLeod. What is Conformity? - Simply Psychology, 2007.

[16] J.-B. Michel, Y. K. Shen, A. P. Aiden, A. Veres, M. K. Gray, J. P. Pickett, D. Hoiberg, D. Clancy, P. Norvig, J. Orwant, et al. Quantitative analysis of culture using millions of digitized books. Science, 331(6014):176-182, 2011.

[17] M. Natale. Convergence of mean vocal intensity in dyadic communication as a function of social desirability. Journal of Personality and Social Psychology, 32(5):790, 1975.

[18] M. Natale. Social desirability as related to convergence of temporal speech patterns. Perceptual and Motor Skills, 40(3):827-830, 1975.

[19] B. Reeves and C. Nass. The Media Equation: How People Treat Computers, Television, and New Media Like Real People and Places (Center for the Study of Language and Information Publication Lecture Notes). Center for the Study of Language and Inf, 2003.
[20] G. Shaw-Garlock. Looking forward to sociable robots. International Journal of Social Robotics, 1(3):249-260, 2009.

[21] M. Sherif. A study of some social factors in perception. Archives of Psychology (Columbia University), 1935.

[22] J. Webb. Studies in dyadic communication: Proceedings of a research conference on the interview. Pergamon, pages 115-133, 1970.

[23] E. J. Weiner and W. Labov. Constraints on the agentless passive. Journal of linguistics, 19(1):29-58, 1983.

[24] Worldrobotics.org. World Robotics - Industrial Robots 2012 Executive Summary. Technical report, 2012. 